\title{
Effect of Mycobacteria M1 on Phytoremediation of Polycyclic Aromatic Hydrocarbons by Ryegrass in Contaminated Soil
}

\author{
$\mathrm{Ru} \mathrm{Li}^{1, \mathrm{a}}$, Xuan Gong ${ }^{2, \mathrm{~b}}$ Zongqiang Gong ${ }^{3, \mathrm{c}}$ \\ ${ }^{1}$ Northeastern University. No.3-11, Wenhua Road, Heping District, Shenyang, P, R, China. \\ ${ }^{2}$ Northeastern University. No.3-11, Wenhua Road, Heping District, Shenyang, P, R, China. \\ ${ }^{3}$ Institute of Applied Ecology. Chinese Academy of Science. No. 72, Wenhua Road Shenhe District \\ Shenyang, P, R, China \\ aliru@mail.neu.edu.cn, bgongxuan@mail.neu.edu.cn, 'czgong@iae.ac.cn
}

\begin{abstract}
Keywords: polycyclic aromatic hydrocarbons; rhizosphere effect; microbe-assisted remediation; dominant degradation bacteria

Abstract. The microbe-assisted remediation has been recognized as a potentially effective method for the removal and degradation of organic contaminants in soils. A study was conducted to examine the effects of inoculation with Mycobacteria M1 on phytoremediation of polycyclic aromatic hydrocarbons (PAHs) by ryegrass in contaminated soil. The results showed that Mycobacteria M1 had the ability to degrade PAHs. Ryegrass exudates can promote the degradation of PAHs by Mycobacteria M1. The group which added $80 \mathrm{mg} \cdot \mathrm{L}^{-1}$ ryegrass exudates reached the best promoting effect, the degradation rate of phenanthrene and pyrene reached $90.08 \%$ and $81.47 \%$. The general trend in the degradation of phenanthrene was near- rhizosphere $>$ rhizosphere $>$ far-rhizosphere in rhizobox experiment. The results suggest that ryegrass-Mycobacteria M1 association can be used to renovate the contaminated soil by PAHs.
\end{abstract}

\section{Introduction}

Polycyclic aromatic hydrocarbons (PAHs) are the common persistent organic pollutants in soil, it has strong carcinogenicity, mutagenicity and teratogenicity [1]. It brings the serious ecological risk, and it is the urgent problem to repair the polluted soil of polycyclic aromatic hydrocarbons. Bioremediation is one of the important methods for the remediation of PAHs contaminated soils, including microbial remediation and phytoremediation. Co-remediation of PAHs contaminated soil by plant and microorganism has become an important research direction [2]. More than 40 kinds of plants were reported to have the ability to promote the degradation of PAHs, such as poplar, willow, pine, corn, alfalfa and ryegrass and so on [3]. However, due to the low bioavailability of PAHs in soil and the lack of PAHs degrading bacteria and other factors, the remediation of PAHs contaminated soil by pure phytoremediation is difficult [4]. The study showed that PAHs specific degradation bacteria or plant growth promoting bacteria (nodule bacteria, mycorrhizal fungi and so on) can effectively improve the efficiency of phytoremediation [5].

In this study Mycobacteria M1 which is one of the PAHs degrading microorganisms was selected. Ryegrass which have the ability to promote PAHs degradation were used. The aim of the work was to evaluate the potential of the ryegrass-Mycobacteria M1 association to remediate PAHs contaminated soil.

\section{Materials and Methods}

The Collection of the Root Exudates .The roots of ryegrass were washed with distilled water and dipped in solution of $0.5 \mathrm{mmol} / \mathrm{L} \mathrm{CaCl}$ for 4 hours, moved them in the Milli-Q ultra pure water for 24 hours. Ryegrass exudates were collected and filter by the 0.45 um filter membrane. The filter liquor was evaporated and the TOC of concentrates was $522 \mathrm{mg} / \mathrm{L}$.

The Test Soils and Microbial Preparation. The experimental soil was a kind of artificial simulated contaminated soils. The soils without pollution was dried in the air, sifted them through sieves with 2 $\mathrm{mm}$ aperture. Phenanthrene and pyrene was dissolved into the acetone, mixed with $10 \%$ of the soil, 
placed in fume cupboard for 24h. After the acetone volatilized completely, all of soil were mixed, sifted the mixed soil through sieves with $2 \mathrm{~mm}$ aperture, and stored in $4{ }^{\circ} \mathrm{C}$ freezer for $30 \mathrm{~d}$.

Mycobacterium M1 was isolated, purified from the contaminated soils. It was cultured in liquid medium at $30^{\circ} \mathrm{C}, 130 \mathrm{rpm}$. There were $0.5 \mathrm{~g}$ yeast extract, $5.0 \mathrm{~g} \mathrm{NaCl}, 10.0 \mathrm{~g} \mathrm{NH} \mathrm{NNO}_{2}, 5.0 \mathrm{~g} \mathrm{~K}_{2} \mathrm{HPO}_{4}$, $0.2 \mathrm{~g} \mathrm{MgSO}_{4} \cdot 7 \mathrm{HO}_{2}$ and $0.5 \mathrm{~g}$ glucose in $1000 \mathrm{ml}$ water. The $\mathrm{pH}$ was 7.2-7.5.

Experimental design. One control group (only 200g artificial simulated contaminated soil) and four treatment groups were set up. In the treatment groups $1 \mathrm{ml}$ of bacteria liquid which was in the logarithmic phase of growth and ryegrass exudates (the concentration was $0,40,80,160 \mathrm{mg} / \mathrm{kg}$ respectively) were added into $200 \mathrm{~g}$ artificial simulated contaminated soil. After mixing evenly, put each groups into a black plastic cup with small holes at the bottom, then cultured them in constant temperature incubator at $28^{\circ} \mathrm{C}$. In order to keep the moisture and the air permeability of soil, the soil was watered and stirred regularly. The soil samples were collected on the $2^{\text {th }}, 5^{\text {th }}, 10^{\text {th }}, 15^{\text {th }}, 30^{\text {th }}$ day respectively.

Rhizobox Experiment. A rhizobox [6] was used to plant ryegrass. The dimension of the rhizobox was $15 \times 15 \times 12$ (length $\times$ width $\times$ height $\mathrm{cm}$ ). The rhizobox was divided into several sections by nylon cloth (300 mesh). The ryegrass seedlings were transplanted to rhizobox. Mycobacterium M1 was inoculated at $15 \%$ of soil. The soil samples were collected in rhizosphere, near-rhizosphere and far -rhizosphere on the $2^{\text {th }}, 5^{\text {th }}, 10^{\text {th }}, 15^{\text {th }}, 30^{\text {th }}$ day respectively.

The extract and determine of PAHs. $10 \mathrm{ml} \mathrm{15 \%} \mathrm{NaCl,} 20 \mathrm{ml} \mathrm{C}_{3} \mathrm{H}_{6} \mathrm{O}$ and $15 \mathrm{ml} \mathrm{CH}_{2} \mathrm{Cl}_{2}$ were added into the samples. After shaking at the speed of $150 \mathrm{r} \cdot \mathrm{min}^{-1}$ for 16 hours, the supernatant was dried in a fume hood and fixed with $2 \mathrm{ml}$ of methyl alcohol. The concentration of phenanthrene and pyrene were determined by the Agilent 1200 series high performance liquid chromatography (HPLC) and the C-18 chromatographic column(ZORBAX Eclipse). Chromatographic conditions was as follows: column temperature $35^{\circ} \mathrm{C}$, mobile phase of methanol, flow rate of $0.8 \mathrm{ml} \cdot \mathrm{min}^{-1}$, sample quantity for $10 \mathrm{ul}$. The detection wavelength of pyrene was $240 \mathrm{~nm}$ and the phenanthrene was $290 \mathrm{~nm}$.

\section{Results}

The Effect of Ryegrass Exudates and Mycobacterium M1 on degradation of Phenanthrene and Pyrene. As shown in Fig. 1. and Fig. 2. Mycobacterium M1 has good degradation effect on phenanthrene and pyrene, ryegrass exudates promoted the ability of Mycobacterium M1 to degrade phenanthrene and pyrene. The degradation of phenanthrene and pyrene by Mycobacterium M1 presented a tendency of increasing with the increase of incubation time, the phenanthrene and pyrene's degradation rate reached $69.87 \%$ and $50.85 \%$ on the 30th day. It showed that Mycobacterium M1 was an advantage bacterial species of strains of the degradation of PAHs.The difference of degradation rate caused by the different nature of the pollutants. The construction of pyrene is more complex than phenanthrene, so it is more difficult to be degraded. Ryegrass exudates promoted the degradation of phenanthrene and pyrene. The group which added $80 \mathrm{mg} / \mathrm{kg}$ ryegrass exudates reached the best promoting effect, the degradation rate of phenanthrene and pyrene reached $90.08 \%$ and $81.47 \%$ on the 30th day. 


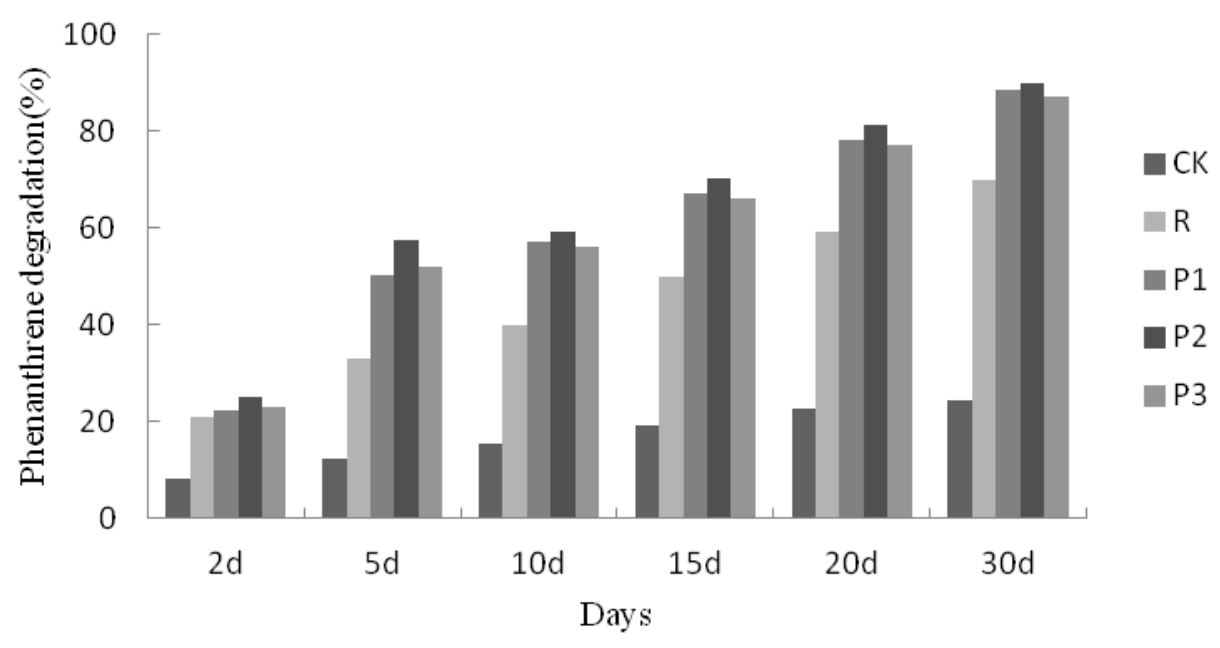

Fig. 1. The effect of ryegrass exudates on degradation of phenanthrene by Mycobacterium M1. CK, contaminated soil by phenanthrene; $R$, contaminated soil inoculated with $\mathrm{M} 1$; P1, P2, P3, contaminated soil with $\mathrm{M} 1$ and 40, 80, $160 \mathrm{mg} / \mathrm{kg}$ ryegrass exudates.

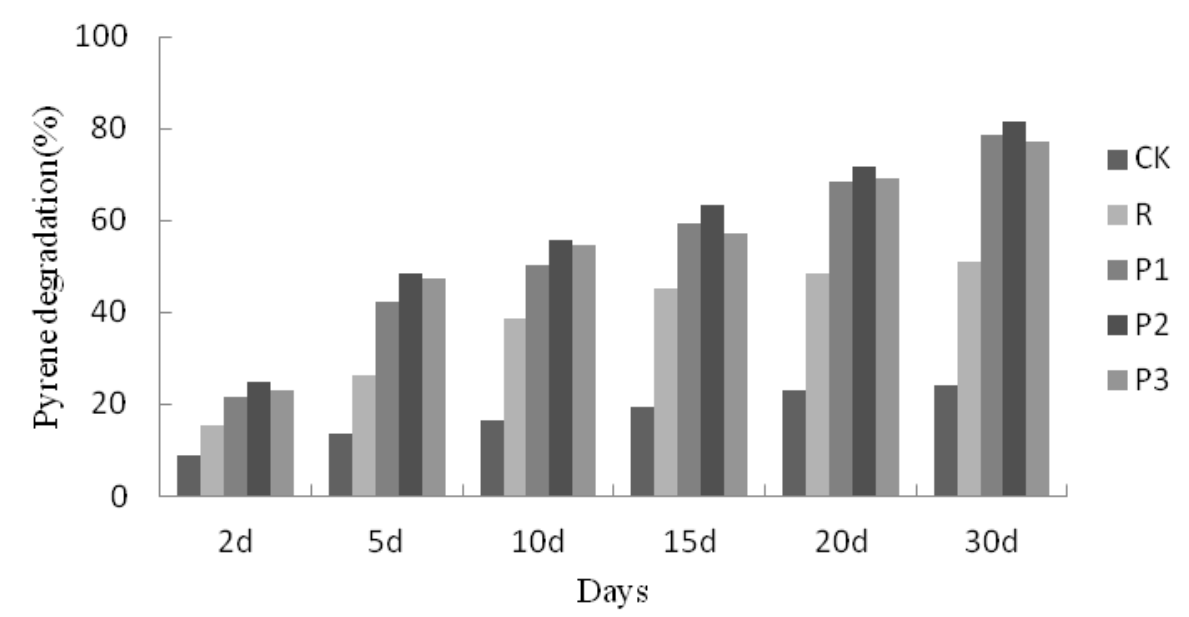

Fig. 2. The effect of ryegrass exudates on degradation of pyrene by Mycobacterium M1. CK, contaminated soil by phenanthrene; $R$, contaminated soil inoculated with $\mathrm{M1}$; P1, P2, P3, contaminated soil with M1 and 40, 80, $160 \mathrm{mg} / \mathrm{kg}$ ryegrass exudates.

The Effect of Ryegrass Exudates on Degradation of Phenanthrene by Mycobacterium M1 in rhizobox. In order to investigate rhizosphere effects on the biodegradation of xenobiotics, a rhizobox experiment was conducted. The degradation of phenanthrene without Mycobacterium M1 was shown in Fig.3. The highest degradation rate of phenanthrene was $80.52 \%$ at near-rhizosphere on the $30^{\text {th }}$ day. The highest degradation rate of phenanthrene with Mycobacterium M1 was $89.74 \%$. (shown in Fig.4.) It was also at near-rhizosphere on the $30^{\text {th }}$ day. The ryegrass-Mycobacterium M1 symbiotic association can increase the degradation of phenanthrene in the soil. The general trend in the degradation of phenanthrene was near- rhizosphere $>$ rhizosphere $>$ far-rhizosphere. 


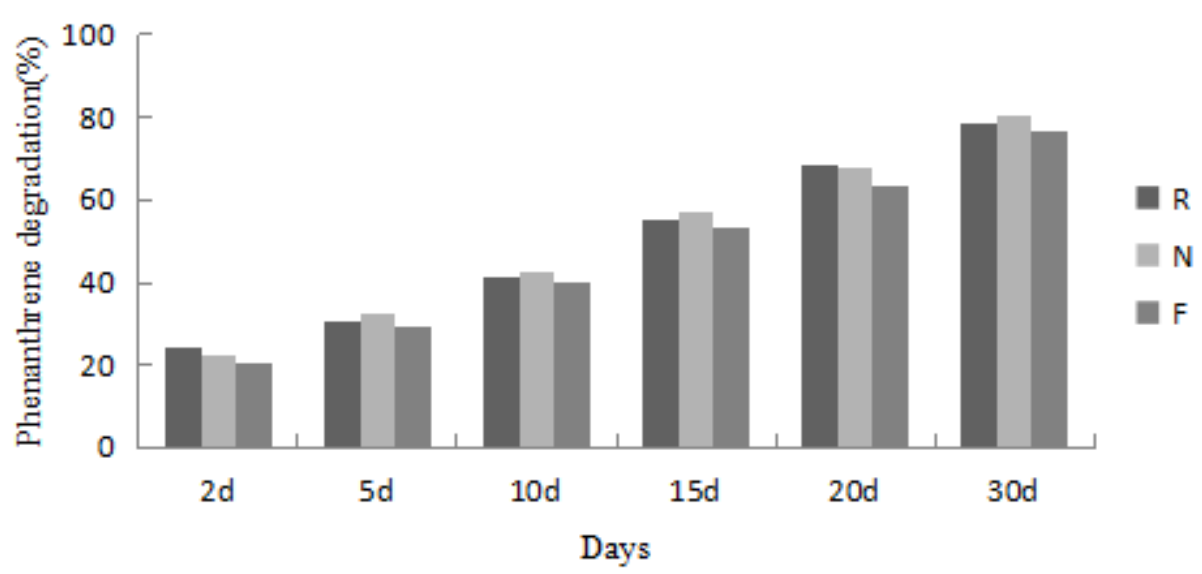

Fig. 3. The rem oval rate of phenanthrene by ryegrass. $R$, rhizosphere; $\mathrm{N}$, near-rhizosphere; $\mathrm{F}$, far-rhizosphere.

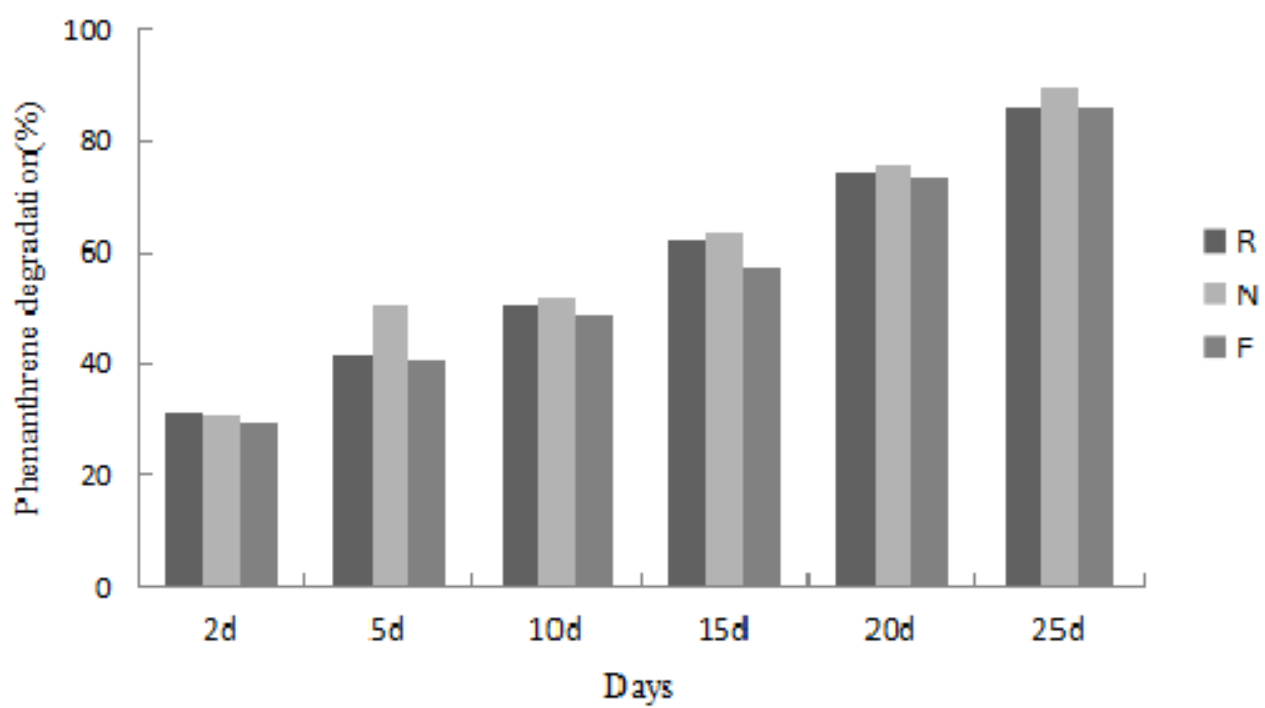

Fig. 4. The removal rate of phenanthrene by ryegrass and Mycobacterium M1. R, rhizosphere; N, near-rhizosphere; F, far-rhizos where.

\section{Discussion}

Microbe-assisted phytoremediation has now emerged as a promising method for removal of soil which was contaminated by PAHs. The different plants have the different effect on PAHs degradation. Some plant species (such as ryegrass, alfalfa and so on) had the obvious promoting effect on the degradation of PAHs[3]. Several bacterial species (such as Thizobia, Arbuscular mycorrhizal fungi, Mycobacterium and so on) are able to utilize PAHs or heterocyclic aromatic compounds [5]. The higher degradation rate of PAHs in this study suggests that ryegrass-Mycobacterium M1 symbiotic association played a role in the remediation of PAH-contaminated soil. Plants can change microbial flora structure, increase the quantity or increase its activity of promoting the degradation of organic pollutants through the rhizosphere effect. The rhizosphere effect associated with plant species, microbial species and the composition and concentration of root exudation. When the concentration of the root exudation exceeds a certain concentration, it will produce an inhibition effect on soil microorganisms, thereby inhibiting the degradation of pollutants[7]. It is necessary to employ suitable combinations of plant species and 
their associated rhizosphere microbes such as plant growth promoting rhizobacteria or contaminant degraders.

\section{Conclusion}

The present study demonstrated that ryegrass can play a significant role in the dissipation of PAHs and inoculation with Mycobacterium M1 enhanced PAHs degradation in the soil.

1. Mycobacterium M1 has good degradation effect on PAHs.

2. Ryegrass exudates promoted the ability of Mycobacterium M1 to degrade PAHs.

3. The ryegrass- Mycobacterium M1 symbiosis may be a suitable plant-microbe partnership for phytoremediation of PAH-contaminated soil.

However, the processes involved in the rhizosphere remain uncertain, and many factors can influence the environment behaviors of xenobiotics in the rhizophere. Further studies are required to elucidate the metabolic pathway of PAHs degradation in plant-microbial associations and molecular feedback mechanisms that lead to PAHs degradation and transformation in the rhizosphere.

\section{References}

[1] Samanta SK, Singh OV, Jain RK: Trends in Biotechnology Vol. 20 (2002), p. 243-248.

[2] Zhao HP, Wu QS, Wang L: Journal of Hazardous Materials Vol. 164 (2009), p. 863-869.

[3] Lee J: Biotechnology and Bioprocess Engineering Vol. 18 (2013), p. 431-439.

[4] Johnson D.L, Anderson D.R: Soil Biology and Biochemistry Vol. 36 (2004), p. 33-38.

[5] Teng Y, Shen Y Y, Luo Y M: Journal of Hazardous Materials Vol. 186 (2011), p. 1271-1276.

[6] Wang Z.W, Shan X.Q, Zhang S.Z: Chemosphere Vol. 46 (2002), p. 1163-1171.

[7] J. Rezek, M. Mackova, F. Zadrazil, T. Macek: Int.J. Phytorem Vol. 11 (2009), p. 65-80. 